\title{
Leiomioma de esôfago com características epidemiológicas anormais: relato de caso
}

\author{
Esophagus leiomyoma with abnormal epidemiological characteristics: case report
}

\author{
Francini de Barrios Pereira', Bruno da Costa Leão ${ }^{2}$, Luiz Fernando Leli ${ }^{3}$, Marcelo de Paula Lima ${ }^{4}$
}

Pereira FB, Leão B, Leli LF, Lima MPL. Leiomioma de esôfago com características epidemiológicas anormais: relato de caso / Esophagus leiomyoma with abnormal epidemiological characteristics: case report. Rev Med (São Paulo). 2018 maio-jun.;97(3):00-00.

RESUMO: Introdução: Apesar da raridade, o leiomioma de esôfago é o tumor benigno não-epitelial mais frequentemente encontrado neste órgão. Relato de Caso: Relata-se o caso de uma paciente de 73 anos diagnosticada, inicialmente, com massa esofágica a esclarecer, oligossintómatica. Realizou-se uma TC de tórax que evidenciou a presença de tumoração em mediastino posterior, proveniente da parede proximal do esôfago de crescimento extraluminal. Foi realizada uma endoscopia digestiva alta com biopsia da lesão, porém essa foi inconclusiva. Optou-se por conduta cirúrgica, através de uma toracotomia direita, sendo o tumor esofágico enucleado e a parede esofágica reconstruída. O anatomopatológico e imuno-histoquímico confirmaram o diagnóstico de leiomioma esofágico. Conclusão: O caso possui características epidemiológicas atípicas, sendo elas a faixa etária, o sexo e localização do leiomioma de esôfago. Além disso, a revisão bibliográfica enfatiza a raridade desta patologia, que geralmente é um achado incidental em exames de imagem ou endoscópicos, sendo um desafio diagnóstico e terapêutico.

Descritores: Leiomioma; Esôfago; Neoplasias/diagnóstico; Doenças do esôfago; Cirurgia torácica.

\begin{abstract}
Introduction: Despite its rarity, the leiomyoma of the esophagus is the most common non-epithelial benign tumor in this organ. Case Report: The case of a 73-year-old woman diagnosed, initially, with esophageal mass to be clarified, oligosynthetics, was reported. A thorax CT scan revealed the presence of a tumor in the posterior mediastinum, coming from the proximal wall of the esophagus with extraluminal growth. A high digestive endoscopy and a biopsy of the lesion were performed, but turned out to be inconclusive. We chose surgical management through a right thoracotomy, with the esophageal tumor enucleated and the esophageal wall reconstructed. The pathology and immunohistokymic confirmed the diagnosis of a esophagealleiomyoma. Conclusion: The case has atypical epidemiological characteristics, such as the age group, the gender and location of the leiomyoma of the esophagus. Moreover, the literature review emphasizes the rarity of this pathology, which is usually an incidental finding in imaging or endoscopic exams, being quite a challenge not only diagnostically, but also therapeutically.
\end{abstract}

Keywords: Leiomyoma; Esophagus; Neoplasms/diagnosis; Esophageal diseases; Thoracic surgery.

Pôster apresentado no III Congresso Internacional de Cirurgia Geral FAMERP/FUNFARME, São José do Rio Preto, SP, 22-24/09/2016. Resumo publicado nos anais do Congresso; Pôster apresentado na IV Jornada Médico Acadêmica da Faculdade de Medicina da Universidade de Franca, Franca, SP, 21/10/2016.

1. Acadêmica do $6^{\circ}$ ano do Curso de Graduação em Medicina da Universidade de Franca. Santa Casa de Franca. ORCID: http:// orcid.org/0000-0001-5220-0125. E-mail: francinidebarrios@hotmail.com.

2. Médico do Serviço de Cirurgia Geral da Santa Casa de Franca. ORCID: https://orcid.org/0000-0002-4060-5202. E-mail: bruno_dcl@hotmail. com.

3. Membro Titular da Sociedade Brasileira de Cirurgia Oncológica e Chefe do Serviço de Residência da Santa Casa de Franca. Orientador. ORCID: https://orcid.org/0000-0002-5589-3753.E-mail: luizleli@yahoo.com.br.

4. Membro Titular da Sociedade Brasileira de Pneumologia e Tisiologia e Diretor Clínica da Santa Casa de Franca. ORCID: https://orcid. org/0000-0002-2928-417X. E-mail: marcelodepaulalima.lima@yahoo.com.br.

Endereço para correspondência: Francini de Barrios Pereira. Rua Hortêncio Mendonça Ribeiro No 856, Prolongamento Jardim Lima. Franca, SP. CEP: 14403-099. E-mail: francinidebarrios@hotmail.com. 


\section{INTRODUÇÃO}

A pesar da raridade, o leiomioma de esôfago é o tumor benigno não-epitelial mais frequentemente encontrado nesse órgão. Representa apenas $0,5-0,8 \%$ dos tumores esofágicos ${ }^{1,2}$. Originam-se da muscular própria, e raramente, da muscular da mucosa. Ocorrem mais frequentemente em homens do que em mulheres na idade entre 20 e 59 anos $^{3}$, sendo raro nas crianças ${ }^{3}$.

A localização preferencial é no segmento médio e inferior do esôfago, com tamanho variável, consistência endurecida, apresentando superfície lisa e regular e, em alguns casos, multilobulada, recoberta por mucosa de coloração normal, sem solução de continuidade, que desliza livremente sobre a lesão, a não ser que apresente erosão ou ulceração apical. A transformação maligna é rara, se de fato ocorre.

Geralmente são assintomáticos, sendo um encontro casual em endoscopias digestivas de rotina ou até que atinjam grande tamanho para apresentar sintomas como disfagia, dor retroesternal, pirose, tosse, odinofagia, perda de peso e sangramento digestivo ${ }^{4}$.

O diagnóstico endoscópico é de presunção, pois se trata de lesão da parede do esôfago, não sendo de diagnóstico endoscópico e, portanto, é limitação do método e não do profissional; e, dificilmente, se consegue firmar um diagnóstico histológico, pois as biópsias não atingem o plano muscular de origem das lesões, exceto em casos onde exista uma ulceração e o tecido muscular se encontre exposto $^{5}$.

Em virtude de sua natureza miogênica, o método de eleição para confirmação do seu diagnóstico é a ultrassonografia endoscópica (USE). Com facilidade, localiza-se a camada muscular própria (quarta camada) do esôfago de onde mais frequentemente se origina o leiomioma, que se apresenta como lesão hipoecogênica e homogênea. Quando se tem dúvida da natureza benigna da lesão miogênica, pode-se realizar o diagnóstico histológico da lesão através de biópsias ecoguiadas ${ }^{5}$.

Em geral o leiomioma não necessita de ressecção, ficando esse tratamento indicado nos casos sintomáticos ou quando houver dúvida sobre a natureza da lesão, sendo realizada enucleação da lesão por toracotomia ou videotoracoscopia ${ }^{6,7}$. A ressecção endoscópica é de difícil execução e com risco de perfuração e hemorragia, ficando reservada apenas quando a origem é da muscular da mucosa (segunda camada do esôfago), bem documentada pela ultrassonografia endoscópica e, às vezes, com ressecção ecoguiada ${ }^{2,8}$.

\section{RELATO DE CASO}

N.R.B, 73 anos, sexo feminino, branca, natural e procedente de Franca, em investigação de pneumonia comunitária, foi visualizado incidentalmente alargamento do mediastino em Raio X de Tórax.

Paciente procurou um pneumologista para investigação do achado radiológico, sendo solicitados uma TC de tórax, e posteriormente uma endoscopia digestiva alta (EDA) para investigação do possível tumor mediastinal. Como sintomatologia, a paciente apresentava queixa eventual de disfagia para alimentos sólidos e episódios de engasgamento, além de tratamento prévio para doença do refluxo gastresofágico.

A tomografia computadorizada evidenciou lesão expansiva com componente predominante de tecido mole com calcificações, comprimindo a luz esofágica no seu terço torácico superior medindo aproximadamente $2,5 \mathrm{~cm}$ por $2,5 \mathrm{~cm}$ por $3,5 \mathrm{~cm}$, não sendo visualizado linfoadenomegalia mediastinal.

Ao exame endoscópico foi evidenciado esôfago com mucosa normal, com compressão extrínseca do esôfago proximal, com extensão de mais ou menos 7,0 cm, não aderente a mucosa esofágica.

Foi realizada a biópsia do esôfago proximal, sendo que ao exame microscópico havia fragmentos de mucosa esofágica de espessura habitual, e no córion havia infiltrado inflamatório linfoplasmocitário irregularmente distribuído.

A decisão de tratamento do cirurgião torácico e da paciente foi a ressecção do tumor. Foi realizada uma toracotomia póstero-lateral direita entre o $5^{\circ}$ e $6^{\circ}$ espaços intercostais, sendo que ao adentrar a cavidade torácica observou-se uma tumoração sólida proveniente da parede do esôfago proximal ao nível da veia ázigos. Após a ligadura da veia ázigos, foi realizada uma dissecção minuciosa do esôfago, e procedida a enucleação total da lesão sem abertura da mucosa esofágica. Após a revisão da hemostasia, foi realizada a sutura da parede esofágica, sem intercorrências.

A peça foi encaminhada ao Serviço de Anatomia Patológica, onde o diagnóstico foi de leiomioma esofágico foi confirmado, através de anatomopatológico e perfilimuno-histoquímico.

No pós-operatório a paciente evolui com quadro de pneumonia nosocomial, sendo realizada antibioticoterapia. Recebeu alta após 10 dias de internação e foi orientada a retornar em consulta ambulatorial para acompanhamento do caso. 


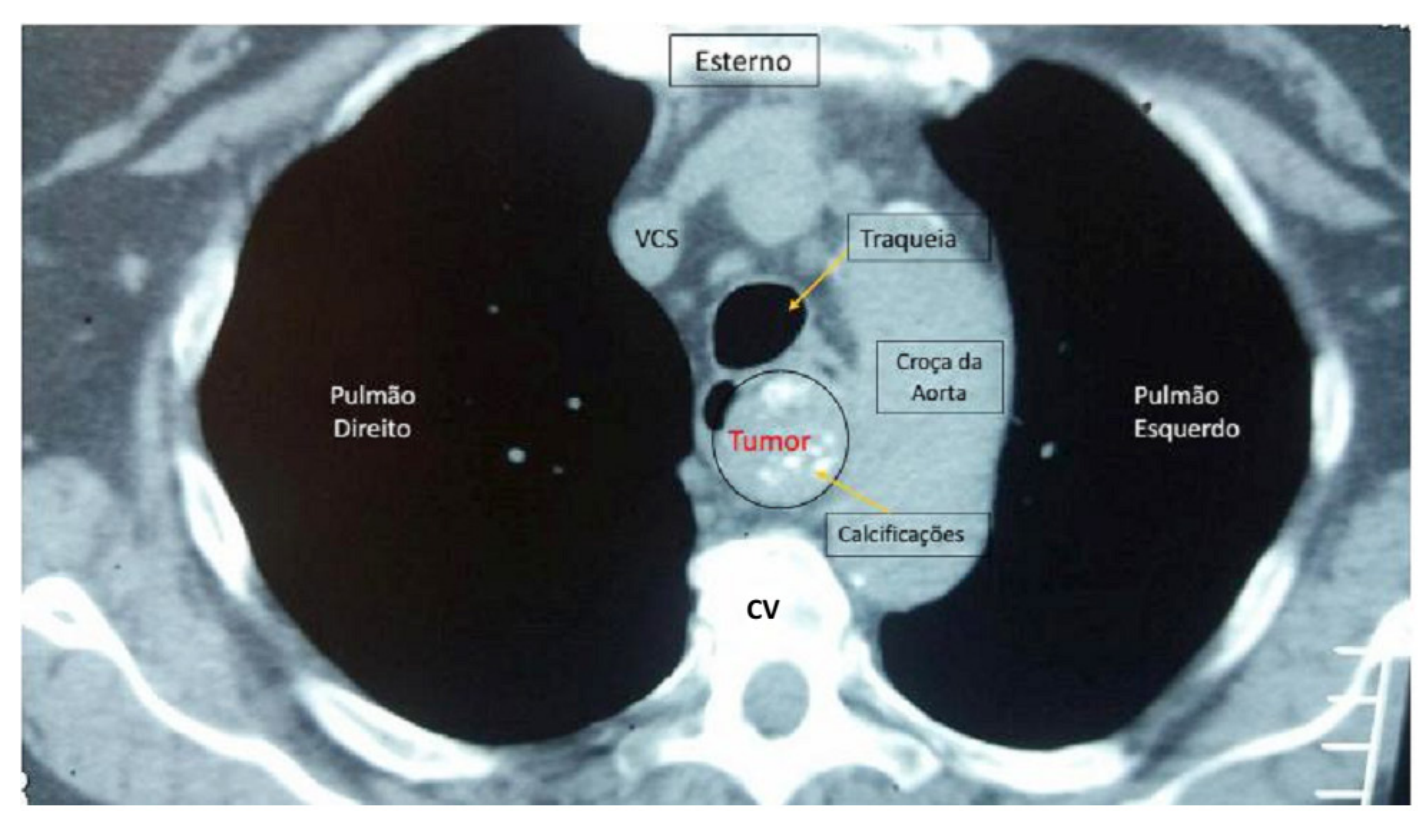

Figura 1. Corte Tomográfico axial evidenciando tumor composto de tecido mole com calcificações, comprimido a luz esofágica

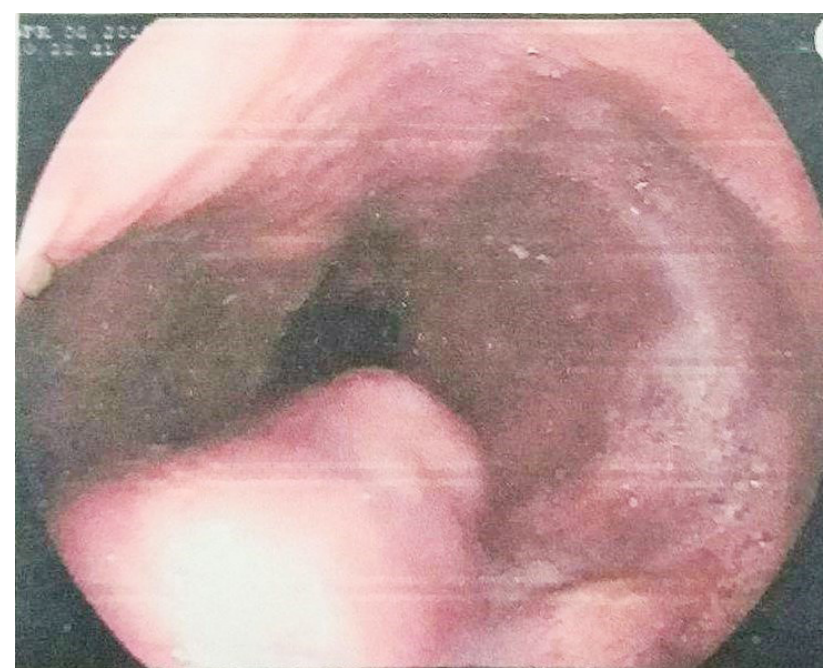

Figura 2. Visão endoscópica da compressão extrínseca em esôfago proximal

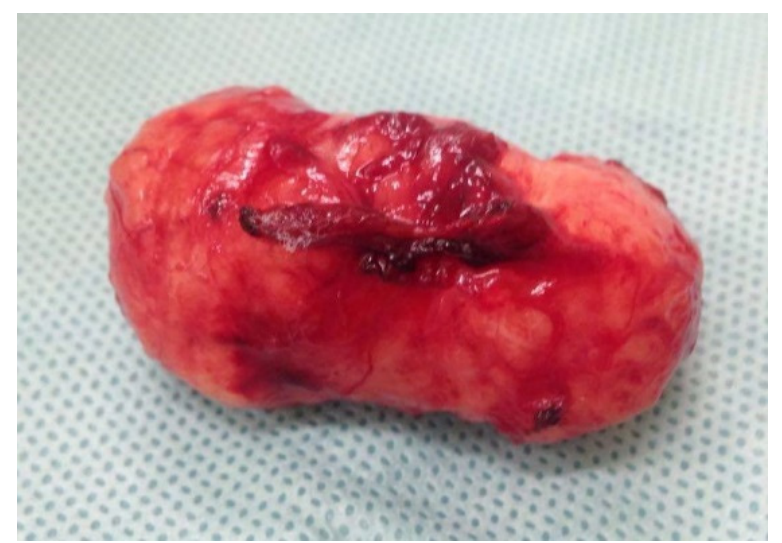

Figura 3. Peça cirúrgica ressecada com dimensões $4,5 \mathrm{~cm}$ por $2,5 \mathrm{~cm}$ por $2,0 \mathrm{~cm}$

\section{DISCUSSÃO}

O leiomioma esofageano é encontrado, em mais de $80 \%$ dos casos, nos terços médio e inferior do esôfago ${ }^{9}$, sendo que no caso em discussão, o leiomioma se localizava no terço proximal do esôfago divergindo da maioria dos casos.

A paciente tinha 73 anos na ocasião do diagnóstico, em discordância com a literatura, já que esse tumor esofágico é mais comum entre os 20 e 59 anos, sendo mais frequente no sexo masculino com frequência relativa de 2:1 a $5: 1^{6}$.

O leiomioma esofágico costuma ser um achado incidental, pois inicialmente não cursa com sintomas ${ }^{6}$. A paciente referiu apresentar sintomatologia de eventual para sólidos e engasgamentos frequentes, porém o diagnóstico da massa mediastinal foi realizado, somente, após a realização de um Raio X tórax que evidenciou um alargamento mediastinal a esclarecer, sendo posteriormente investigado por TC de tórax.

É fato que o diagnóstico de leiomioma esofágico é inicialmente desafiador, visto que os sintomas iniciais são inespecíficos e podem ser confundidos com a Doença do Refluxo Gastroesofágico (DRGE). Os recursos de imagem são muito uteis para guiar o diagnóstico, porém não possuem boa especificidade para o diagnóstico, já que outros tumores benignos esofágicos podem apresentar imagens semelhantes, como fibroma, lipoma, neurilemoma, hemangioma, linfangioma, papiloma escamoso, pólipo fibrovascular e mioblastoma de células granulares; além de malignidades, como carcinomas e tumores do estroma gastrointestinal.

$\mathrm{Na}$ radiografia de tórax, pode apresentar-se como massa mediastinal. Na endoscopia em geral causa discreto 
abaulamento submucoso como ocorreu no caso da paciente, ou redução do lúmen nos exames baritados ${ }^{7}$. A tomografia computadorizada pode mostrar lesão homogênea de tecido mole, intramural ou excêntrica que, raramente, é circunferencial $^{9}$, sendo que no caso da paciente a imagem é circunferencial como pode ser visto na Figura 1. A mucosa frequentemente está normal e a ressonância nuclear magnética pode revelar imagens em T2 com captação sem anormalidades 9 .

A ecoendoscopia (Punção) é usada para definir os limites do tumor e mostra cinco camadas do esôfago: 1) hiperecogênica, mucosa superficial; 2) hipoecogênica, mucosa e muscular da mucosa; 3) hiperecogênica, submucosa; 4) hipoecogênica, muscular própria; 5) hiperecogênica, adventícia ${ }^{7}$.

O diagnóstico definitivo exige análise histopatológica do tumor que se obtém por biopsia préoperatória ou da peça resultante de ato cirúrgico. Entretanto, devem ser evitadas as biópsias o quanto possível; podem ser inconclusivas por escassez de material da submucosa ou originar reação inflamatória que interfere na operação. As dificuldades em relação ao exame anatomopatológico estão exemplificadas no presente caso, pois a biópsia realizada na primeira endoscopia digestiva alta não forneceu material suficiente para esclarecer a natureza histológica da lesão ${ }^{5}$.

O tratamento pode ser cirúrgico ou expectante. A excisão de leiomiomas sintomáticos ou daqueles maiores que $5 \mathrm{~cm}$ é geralmente recomendada. Ao contrário, nos assintomáticos ou menores que $5 \mathrm{~cm}$, pode-se optar por uma conduta expectante, com acompanhamento através de esofagografias periódicas, haja visto que esses tumores têm um aspecto radiológico muito característico, uma velocidade de crescimento geralmente bastante lenta e o risco de degeneração maligna é baixo, porém por escolha da paciente foi decidida a conduta cirúrgica.

No entanto, pela dificuldade de diferenciá-lo da forma maligna, muitos cirurgiões preferem a ressecção do tumor, para posterior encaminhamento ao serviço de anátomo-patologia ${ }^{9}$. Esta foi a conduta tomada pelo nosso serviço, que optou por toracotomia e não por toracoscopia, pela maior segurança na abordagem cirúrgica aberta pela equipe em questão. $\mathrm{O}$ ato cirúrgico foi realizado por um cirurgião torácico e um cirurgião oncológico, sendo que de acordo com a literatura, os resultados são excelentes, não havendo relatos de recorrências 9 .

\section{CONCLUSÃO}

O caso relatado possui características epidemiológicas e topográficas divergentes dos casos relatados de leiomioma esofágico.

Além disso, através da revisão bibliográfica ressaltase a raridade do leiomioma de esôfago, que é considerado um desafio diagnóstico, por ser inicialmente assintomático, na maioria dos casos, sendo geralmente um achado incidental em exames radiológicos ou endoscópicos.

Portanto, o caso relatado promove o conhecimento de um diagnóstico raro, cuja incidência é baixa, e estimula a resolução de desafios diagnósticos e o conhecimento das diferentes formas de manuseio e resolução dos casos.

Contribuição dos autores: Pereira $F B$ - autora e redatora do artigo, pesquisa bibliográfica, edição textual e de imagens, organização de documentação e contato com Comitê de Ética e Plataforma Brasil; Leão BC - Revisão textual; Lelli $L F$ Revisão textual e orientação técnica; Lima $M P$ - Revisão textual, orientação técnica e documentação de comitê de ética.

\section{REFERÊNCIAS}

1. Choong CK, Meyers BF. Benign esophageal tumors: introduction, incidence, classification and clinical features. Semin Thorac Cardiac Surg. 2003;15:3-8.

2. Rice TW. Benign esophageal tumors: esophagoscopy and endoscopic esophageal ultrasound. Semin Thorac Cardiovasc Surg. 2003;15:20-6.

3. Boourque MD, Spigland N, Bensoussan AL, Collin PP, Saguem MH, Brochu P, Blanchard H, Reinberg O. Esophageal Leiomyoma in children: two case reports and review of the literature. J Pediatric Surg. 1989;24:1103-7.

4. Furtado WS, Mello DACPG, Santos VM, Junior WPO, Schroff WLA. . Leiomioma de esôfago tratado por via endoscópica em mulher de 41 anos etários Brasília Med. 2012;49(4):2848. [citado 17 set. 2017]. Disponível em: http://livrozilla.com/ doc/805209/arquivo-em-pdf.

5. Albuquerque W, Rocha LCM, Faria KB. Tumores benignos de esôfago. In: SOBED - Sociedade Brasileira de Endoscopia Digestiva. Endoscopia diagnóstica e terapêutica. São Paulo: SOBED; s.d. Cap.28, p.219 [citado 17 set. 2017]. Disponível em: http://www.gastrointestinalmed.com.br/downloads/ Tumores_Benignos_esofago.pdf.

6. Luh SP, Hou SM, Fang CC, Chen CY. Video-thoracoscopic enucleation of esophageal leiomyoma. World J Surg Oncol. 2012;10:52. http://doi.org/10.1186/1477-7819-10-52.

7. Ray S, Saluja SS, Gupta R, Chattopadhyay TK. Esophageal leiomyomatosis - an unusual cause of pseudoachalasia. Can J Gastroenterol. 2008;22(2):187-9. http://doi.org/10.1186/14777819-10-52.

8. Benedetti G, Sablich R, Bonea M, Marius S.. Fiberoptic endoscopic resection of symptomatic leiomyoma of the upper esophagus. Case report. Acta Chir Scand. 1990;156:807-8.

9. Yang PS, Lee KS, Lee SJ, Kim TS, Choo IW, Shim YM, et al. Esophageal leiomyoma: radiologic findings in 12 patients. Korean J Radiol. 2001;2(3):132-7. https://doi.org/10.3348/ kjr.2001.2.3.132.

Submetido em: 15.11 .17

Aceito em: 26.02.18 